\title{
Análise de demanda em Medicina de Família no Brasil utilizando a Classificação Internacional de Atenção Primária
}

\author{
Analysis of demand for family medical care in Brazil \\ using the International Classification of Primary Care
}

Gustavo de Araújo Porto Landsberg ${ }^{1}$

Leonardo Cançado Monteiro Savassi ${ }^{2}$

André Bonamigo de Sousa ${ }^{3}$

Janaína Miranda Rocha de Freitas ${ }^{3}$

Janaína Le Sann Nascimento ${ }^{3}$

Rafael Azagra ${ }^{4}$

${ }^{1}$ Faculdade de Medicina, Universidade José Rosário Vellano (Unifenas-BH) Rua Líbano 66, Itapoã. 31710-030 Belo Horizonte MG.

gustavolandsberg@gmail.com

${ }^{2}$ Departamento de Ciências Médicas, Universidade

Federal de Ouro Preto.

${ }^{3}$ Residência de Medicina de

Família e Comunidade,

Hospital Regional de Betim.

${ }^{4}$ Departamento de

Medicina, Universitat

Autònoma de Barcelona.

\begin{abstract}
In various countries, motives for contact of patients with Primary Health Care (PHC) is classified by the International Classification of Primary Care (ICPC-2). This instrument enables the assessment of why people seek care, thereby assisting in planning strategies to attend the population's health needs. The scope of this study was to identify the main reasons for same-day appointments in PHC units of a medium-sized Brazilian city. The methodology used was to examine all records of a typical month of three family practice residents. Data were extracted from a secondary database of same-day appointments during the spring of 2010, classified with ICPC and then statistically analyzed. 1222 records were considered; 32 motives accounted for $50 \%$ of contacts. Most common motives were related to the General and Unspecific chapter of the ICPC. About $20 \%$ of visits occurred for administrative reasons. Female sex and greater age were determinants of greater motives for consultation. Knowing the motives for appointments by gender and age may help PHC teams in tackling health problems at the critical point of access to PHC.
\end{abstract}

Key words Primary Health Care, Access to health services, Care, Health service needs and demands
Resumo Em vários países, motivos de contatos entre pacientes e a Atenção Primária (APS) são codificados pela Classificação Internacional da Atenção Primária (CIAP-2). Tal instrumento permite avaliar as razões que levam pessoas a buscarem cuidados, sendo útil no planejamento de estratégias para contemplar as necessidades de saúde da população. Objetivo: estudar os principais motivos de consulta na demanda espontânea da atenção primária em município de médio porte brasileiro. Metodologia: considerou-se todos os registros de acolhimento num mês típico de trabalho de três médicos residentes de medicina de família e comunidade. Os dados foram extraídos de banco de dados secundário, classificados com a CIAP-2 e analisados estatisticamente. Resultados: avaliou-se 1222 registros de consultas; 32 motivos representaram $50 \%$ dos contatos. As razões mais frequentes de busca por cuidados relacionavamse ao capitulo Geral e inespecífico. Quase 20\% das consultas ocorreram por motivos administrativos. Sexo feminino e aumento da idade determinaram maior número de motivos para consultar. Conclusão: conhecer os motivos de consulta por gênero e idade pode ajudar as equipes no enfrentamento dos problemas de saúde apresentados no acolhimento - ponto crítico do acesso à APS.

Palavras-chave Atenção Primária à Saúde, Acesso aos serviços de saúde, Acolhimento, necessidades e demandas de serviços de saúde 


\section{Introdução}

A Atenção Primária a Saúde (APS) tem o desafio de lidar com pessoas, independente de faixa etária, gênero, ciclo de vida ou afecção orgânica. Neste contexto, lida com problemas de saúde que se mostram em um estágio mais inicial, ou indiferenciado, não chegando a se constituir em um diagnóstico formal. Muitos sintomas nunca chegarão a definir uma doença que seja classificável ou codificável ${ }^{1}$.

$\mathrm{Na}$ Conferência Internacional de Alma Ata, em 1978, a Organização Mundial de Saúde (OMS) e a World Organization of National Colleges, Academies and Academic Associations of General Practitioners/Family Physicians (WONCA) concluíram que construir sistemas de cuidados primários adequados dependia do acesso dos profissionais de atenção primária às informações certas, por novos sistemas de classificação ${ }^{2-}$ 3. Um Grupo de Trabalho da OMS desenvolveu então uma Classificação Internacional de Motivos de Consulta em Atenção Primária, chamada "Classificação de Motivos de Consulta" (CMC) ${ }^{4}$ ${ }^{6}$, que serviria de base para o posterior desenvolvimento da Classificação Internacional de Atenção Primária (CIAP ou ICPC, do termo em inglês International Classification in Primary Care), publicada em 1987. Esta classificação está em sua segunda versão, a de19987.

A Classificação Internacional da Atenção Primária (CIAP) trabalha com o conceito de "episódio de cuidado", definido como todo tipo de atenção prestada a um determinado indivíduo que apresente um problema de saúde, desde a primeira vez que a pessoa procura atenção devido àquele problema até o último encontro com o profissional por este motivo. Um episódio de cuidado pode durar uma consulta ou mais, e pode até mesmo nunca se encerrar - como no caso de um paciente com diabetes ou com má formação congênita, por exemplo ${ }^{8}$.

A CIAP tem como premissa registrar três aspectos do episódio de cuidado: o motivo da consulta, o diagnóstico do problema percebido pelo profissional de saúde e a intervenção resultante.

O motivo da consulta (ou razão para o encontro) é um elemento chave no registro, devendo estar claro entre o paciente e o profissional, e ser classificado tal como expresso pelo paciente. Pessoas podem buscar atenção por diversos motivos: uma solicitação burocrática, um anseio, o medo de uma doença, a procura por uma informação técnica, ou a necessidade de discutir situações ligadas a questões não biomédicas - além de sinais, sintomas e doenças pré-estabelecidas (Figura 1).

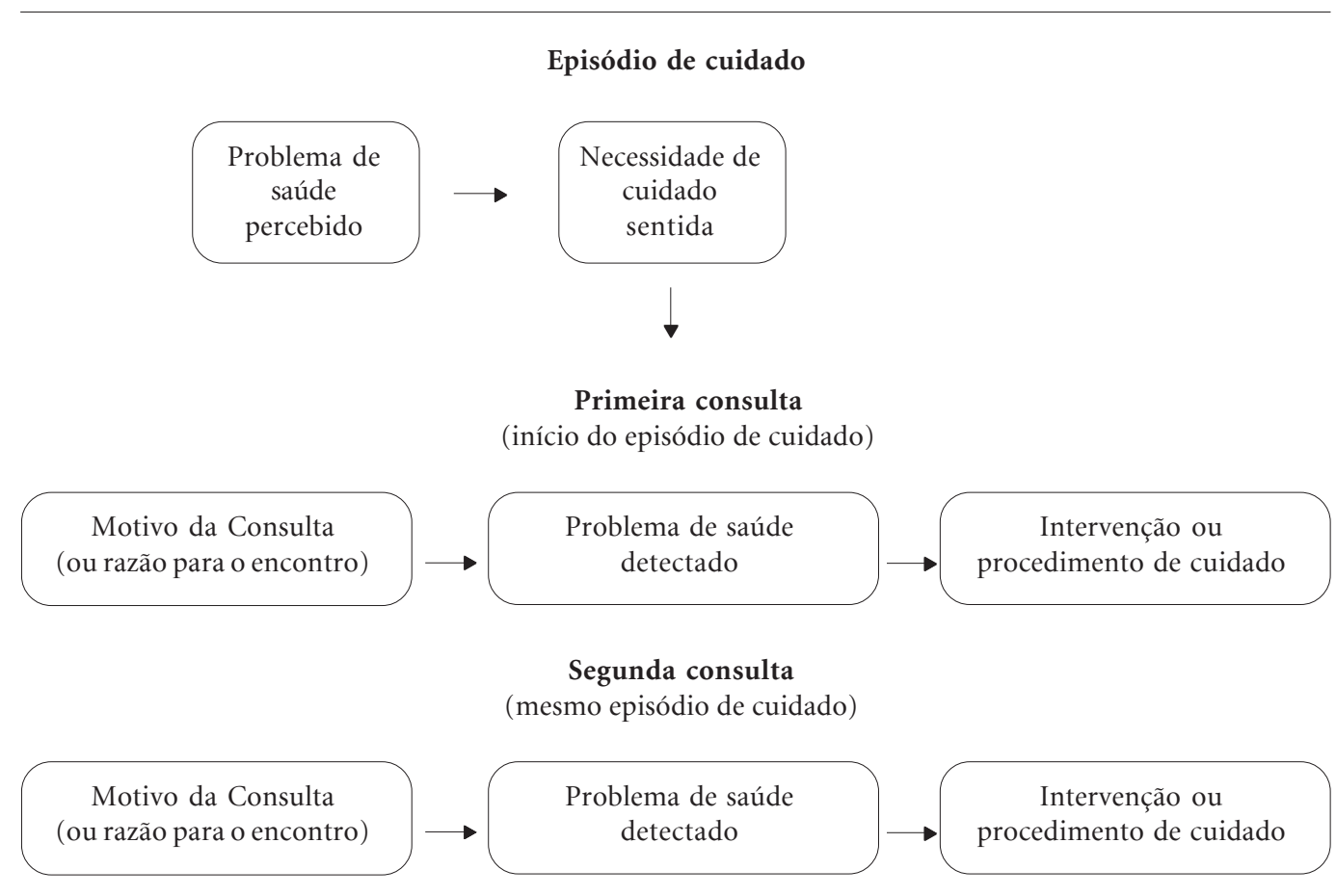

Figura 1. Estrutura do episódio de cuidado. 
A CIAP representa-se com um código alfanumérico de uma letra e dois numerais - tal como a CID. Ela se divide em dezessete capítulos, que abordam todos os sistemas, incluindo o psicológico, a gravidez e o planejamento familiar, problemas sociais e um capítulo de situações gerais e não específicas. Cada capítulo é por sua vez dividido em sete componentes: de queixas e sintomas, de procedimentos e diagnósticos preventivos, de medicações, tratamentos e terapêutica, de resultados de exames, componente administrativo, de acompanhamento e outros motivos de consultas e de diagnósticos e doenças, estas divididas em infecciosas, neoplasias, lesões, anomalias congênitas e outras. O Brasil adquiriu a licença para utilizar a CIAP-2 em 2006 (Figura 2).

A CIAP foi idealizada para contemplar os motivos de consulta, problemas e as intervenções mais frequentes no âmbito da Atenção Primária, sendo, portanto, o sistema de classificação mais adequado para o uso neste nível de atenção. Inclui cerca de 200 sintomas/queixas e 300 diagnósticos comuns em APS. Quase todos apre- sentam uma incidência de pelo menos 1 para cada 1000 pacientes/ano 9 .

Ela não representa uma proposta de substituição à CID, que permanece válida há mais de um século para denominar e classificar causas de morbi-mortalidade. CIAP e CID podem, inclusive, ser utilizadas de maneira concomitante. Um sistema de conversão está disponível e pesquisas empíricas confirmam que ambas são complementares ${ }^{3}$.

Entretanto, os problemas de saúde observados na APS têm características peculiares, já bem estudadas. Em 1963, em seu clássico estudo, Crombie observou que em apenas 50\% das consultas ocorridas neste nível de cuidados é possível estabelecer um diagnóstico preciso ${ }^{10}$. Neste estudo, o autor apresenta cinco conclusões, que permanecem atuais:

1. Muitos "problemas" são autolimitados e requerem apenas observação ativa.

2. Muitos pacientes demandam apenas serviços administrativos.

3. A fisiopatologia de base de "problemas" indefinidos e não graves é desconhecida.

\begin{tabular}{|c|c|c|c|c|c|c|c|c|c|c|c|c|c|c|c|c|c|}
\hline Capítulos & $\begin{array}{l}\vec{\pi} \\
\text { ज्ञ } \\
\ll\end{array}$ & 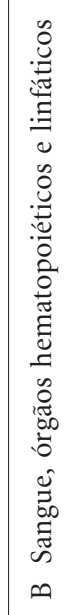 & 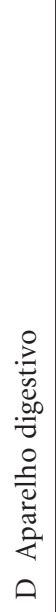 & $\begin{array}{l}\mathscr{0} \\
\stackrel{0}{0} \\
\text { L }\end{array}$ & 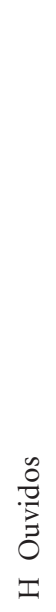 & 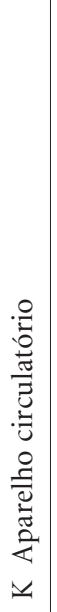 & 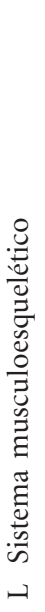 & 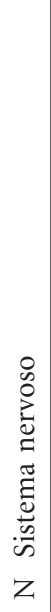 & 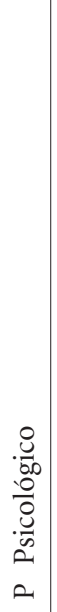 & 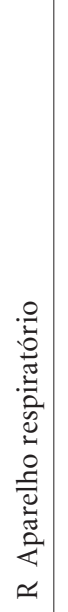 & $\begin{array}{l}\frac{0}{0} \\
\infty \\
\infty\end{array}$ & 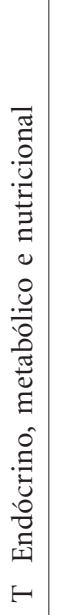 & 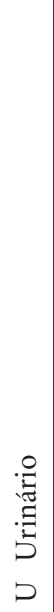 & 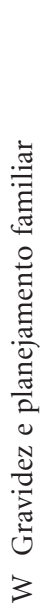 & 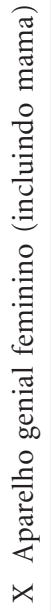 & 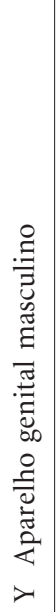 & 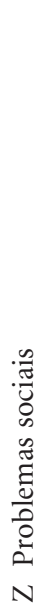 \\
\hline \multirow{7}{*}{ 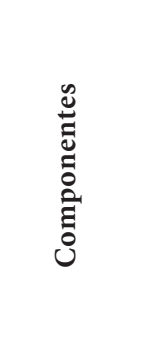 } & \multicolumn{17}{|c|}{1 Queixas e sintomas } \\
\hline & \multicolumn{17}{|c|}{2 Procedimentos diagnósticos e preventivos } \\
\hline & \multicolumn{17}{|c|}{3 Medicações, tratamentos e procedimentos terapêuticos } \\
\hline & \multicolumn{17}{|c|}{4 Resultados de exames } \\
\hline & \multicolumn{17}{|c|}{5 Administrativo } \\
\hline & \multicolumn{17}{|c|}{6 Acompanhamento e outros motivos da consulta } \\
\hline & \multicolumn{17}{|c|}{7 Diagnósticos e doenças } \\
\hline
\end{tabular}

Figura 2. Estrutura biaxial da CIAP-2. 
4. Exames laboratoriais e a tecnologia não colaboram para o complexo processo diagnóstico de "problemas" frequentes.

5. É má prática tentar vigorosamente a definição de um diagnóstico para um "problema" vago, especialmente se este está acompanhado de componentes psicológicos.

Espera-se que uma equipe de APS bem estruturada resolva entre 80 e $95 \%$ dos problemas de saúde de sua população, já que cerca de 50 diagnósticos mais comuns respondem por mais da metade da demanda das unidades básicas de saú$\mathrm{de}^{11}$. Em Porto Alegre, de 7849 atendimentos, 91\% foram resolvidos por Médicos de Família e Comunidade (MFC), sem a necessidade de encaminhamentos para outros níveis do sistema ${ }^{12}$. Em Florianópolis, 2008, de 5698 encontros avaliados, 753 encaminhamentos a outras especialidades médicas foram realizados - ou seja, cerca de 13,2\%. Além disso, 305 foram referidos a outros profissionais de saúde, não-médicos $(5,3 \%)^{13}$.

Segundo Starfield, apenas 26 problemas de saúde correspondem a $50 \%$ de todos os motivos de consultas de médicos de família nos Estados Unidos, número ligeiramente superior aos 22 motivos de consulta para especialistas em adultos não grávidos (medicina interna), embora bem superior aos sete motivos para pediatras, e apenas três para ginecologistas. Além disto, os mesmos dados nos EUA, de 1994, apontam que os MFC encaminham em média apenas $6,3 \%$ dos seus pacientes ${ }^{14}$. Em Florianópolis, de 5698 encontros avaliados, os 30 problemas mais comuns de saúde representaram $50 \%$ de todas as consultas ${ }^{13}$.

Entretanto, para que a Atenção Primária possa exercer seu potencial resolutivo, inicialmente deve-se contemplar o primeiro atributo essencial que caracteriza este nível de cuidados. É função da APS ser a porta de entrada de um sistema de saúde, oferecendo aos usuários acesso ao mesmo ${ }^{14}$.

Dificuldades relacionadas ao acesso à APS existem no mundo todo ${ }^{15-17}$. No Brasil não seria diferente. A ampliação de ferramentas de acesso é pauta de discussão em todos os âmbitos - gestão, academia, controle social, sociedades de especialidades, grupos virtuais - e tem como novo capítulo a recente utilização da escala de risco de Manchester na Atenção Primária do estado de Minas Gerais ${ }^{18}$.

Neste contexto de escassa informação sobre o tema, desenhou-se o presente estudo objetivando determinar os motivos de consulta mais comuns em uma população de médio porte brasileira.

\section{Material e métodos}

Estudo descritivo transversal realizado em três Unidades Básicas de Saúde da Família (UBSF) de Betim, Minas Gerais, Brasil - representando 10\% das equipes do município.

Foi selecionada uma mostra de conveniência em três das equipes sob a responsabilidade do programa de residência em Medicina de Família e Comunidade do Hospital Regional de Betim. Incluiu-se no estudo toda a população atendida na demanda espontânea do mês de outubro de 2010. Os médicos que participaram da coleta de dados eram residentes, cada um responsável por uma equipe de saúde da família. Todos receberam um treinamento constituído por quatro horas de aula teórica e outras quatro horas de treinamento prático em classificação com CIAP-2.

Foram consultados bancos de dados secundários (registros de acolhimento) durante todo o mês de outubro de 2010. Não entraram no estudo as consultas programadas. Posteriormente, os motivos de consulta foram codificados para a Classificação Internacional da Atenção Primária (CIAP-2). A codificação dos residentes foi comparada àquela feita às cegas pelo supervisor do treinamento. Nos casos em que houve disparidades, foram aceitas as codificações do supervisor.

Por se tratar de consulta a banco de dados secundários, não foram aplicados termos de consentimento livre e esclarecido.

\section{Análise dos Dados}

As variáveis coletadas para este estudo foram descritas com a média como medida de tendência central, no caso de variáveis quantitativas, e com a frequência absoluta no caso da qualitativas. Os parâmetros de interesse foram estimados mediante um intervalo de confiança correspondente. Também se estudou a associação entre duas variáveis. Por tratar-se de uma variável qualitativa e uma quantitativa, a associação foi avaliada mediante uma prova T-Student ou ANOVA (para múltiplas variáveis). Todos os intervalos foram realizados com uma confiança de 95\% e todas as hipóteses para comprovar associação de variáveis realizadas com um risco alfa de $5 \%$. Os dados foram analisados com o programa SPSS 17.0 para Mac OS. 


\section{Resultados}

Foi classificado um total de 1.220 registros de motivo de consulta em acolhimento, relativos ao atendimento de 562 pessoas - das quais $68,1 \%$ pertenciam ao sexo feminino e $31,9 \%$ ao masculino. O Gráfico 1 representa a distribuição por gênero e faixa etária da população estudada.

No total, 305 diferentes motivos para consulta foram codificados na amostra. Os 50 mais frequentes destes contatos são explicitados na Tabela 1.

Os dois motivos de consulta mais frequentes estão relacionados com resultados de exames e prescrição de medicação, com 11,3\% do total. Em terceiro lugar está o motivo clínico mais frequente: a cefaleia. Seguem então o quarto e quinto motivo de consulta - febre e tosse, respectivamente.

Os 32 primeiros motivos concentram cerca de $50 \%$ das consultas. Destes, 7 são administrativos e 25 são identificados como clínicos (como dor ou outros sintomas). Praticamente $20 \%$ das consultas ocorreu por razões administrativas.

$\mathrm{Na}$ Tabela 2 se observa que quase 30\% dos motivos de consulta estão classificados no capítulo A da CIAP-2, relativo a problemas gerais ou inespecíficos. A partir daí, seguem os motivos de consulta respiratórios, digestivos, musculoesqueléticos, de pele e neurológicos - que somam 36,5\% do total. Aproximadamente um terço das razões para consultar-se estão classificados nos demais capítulos.
Em 44\% das consultas, os pacientes apresentaram apenas um motivo para procurar atendimento. Outros $24,2 \%$ tinham duas razões para buscar uma consulta, e ainda 31,8\% apresentavam 3 ou mais motivos.

A média de motivos de consulta por pessoa aumenta com a faixa etária até os 59 anos (Gráfico 2), embora este número se reduza ligeiramente a partir dos 60 anos. A média passa de 1,87 motivos por pessoa na faixa etária da infância para até 2,40 na faixa de 50 a 59 anos. Os achados foram estatisticamente significativos quando comparadas a médias de adultos jovens (18-39 anos) com a de adultos entre 40 a 59 anos $(\mathrm{p}=0,01)$.

Houve diferença estatística entre gêneros quanto ao número de queixas por pessoa. Homens apresentaram uma média de 1,98 motivos de consulta; as mulheres, 2,23 ( $\mathrm{p}=0,03)$. Há também uma grande diferença de proporção de atendimento entre os gêneros, que chega a 8,2 mulheres para cada homem atendido na faixa dos 30-39 anos (Gráfico 3).

Quando analisadas as médias de motivos de consulta distribuída pelos dias da semana, observa-se que na segunda-feira e na sexta-feira os pacientes tendem a apresentar mais motivos de consulta. Tais achados são estatisticamente relevantes quando se compara a média de sexta-feira com as médias de terça e a quarta-feira $(\mathrm{p}<0,05)$.

Os motivos "Gerais e inespecíficos" foram os responsáveis mais frequentes pelos contatos com

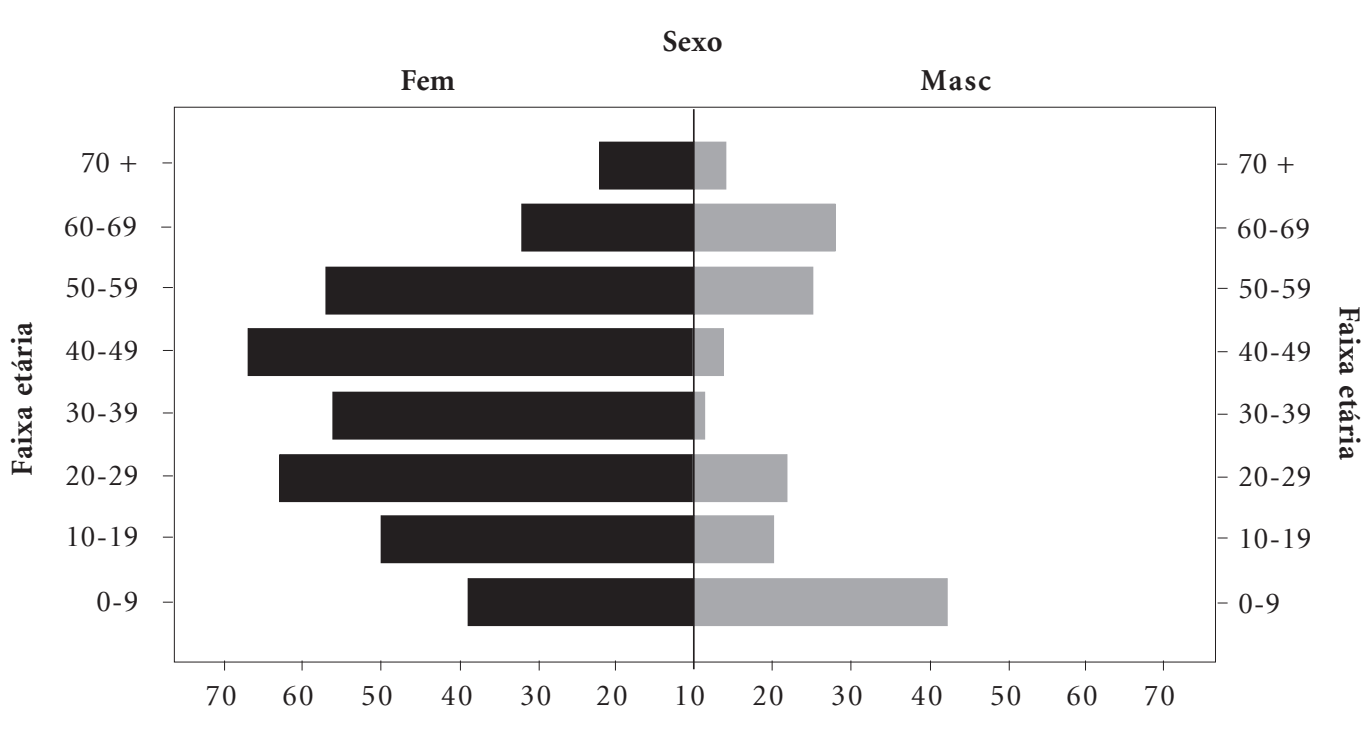

Gráfico 1. Distribuição por gênero e idade da população estudada. 
Tabela 1. Registro dos 50 motivos mais prevalentes de contatos na atenção primária classificados pela CIAP-2, Betim, 2010.

Codificação pela CIAP-2

1. A60 - Resultado de Análises / Procedimentos

2. A50 - Medicação/prescrição/renovação/injeção

3. N01 - Cefaleia

4. A03 - Febre

5. R05 - Tosse

6. A01 - Dor Generalizada/Múlipla

7. K50 - Medicação/prescrição/renovação/injeção (sistema Circulatório)

8. P50 - Medicação/prescrição/renovação/injeção (sistema Psicológico)

9. L14 - Sinais/sintomas da coxa/perna

10. A85 - Efeito adverso de fármaco dose correta

11. L03 - Sinais/sintomas da região lombar

12. S02 - Prurido

13. A04 - Debilidade/cansaço geral/fadiga

14. R03 - Respiração ruidosa

15. D01 - Dor abdominal generalizada/cólicas

16. D10 - Vômito

17. F05 - Outras perturbações visuais

18. R02 - Dificuldade respiratória, dispneia

19. S04 - Tumor/inchaço localizado

20. U01 - Disúria/micção dolorosa

21. R21 - Sinais/sintomas da garganta

22. F01 - Dor no olho

23. Z10 - Problema relacionado com sist. de saúde

24. P01 - Sensação ansiedade/nervosismo/tensão

25. A05 - Sentir-se doente

26. A16 - Criança irritável

27. K60 - Resultado de análises/procedimentos (sistema circulatório)

28. K85 - Pressão arterial elevada

29. R25 - Expectoração/mucosidade anormal

30. T50 - Medicação/prescrição/renovação/injeção (sistema Endócrino/Metabólico)

31. X14 - Secreção Vaginal

32. $\mathrm{X} 15$ - Sinais/sintomas da vagina

33. A11 - Dores torácicas NE

34. A13 - Receio/Medo do tratamento

35. A62 - Procedimento administrativo

36. A65 - Episódio/problema iniciado por outro NE

37. D02 - Dores abdominais, epigástricas

38. N17 - Vertigens/tonturas

39. Z05 - Problemas com condições de trabalho

40. A34 - Análise de sangue

41. A48 - Esclarecimento/discussão do motivo da consulta

42. A49 - Outros procedimentos preventivos

43. A72 - Varicela

44. D20 - Sinais/sintomas da boca/língua/lábios

45. F03 - Secreção ocular

46. F29 - Outros sinais/sintomas oculares

47. S05 - Tumores/inchaços generalizados

48. S06 - Erupção cutânea localizada

49. A92 - Alergia/reação alérgica NE

50. T03 - Perda de apetite

51. U07 - Outros sinais/sintomas urinários
Frequência Percentual Percentual

cumulativo

$\begin{array}{rrr}91 & 7.7 & 7.7 \\ 42 & 3.6 & 11.3 \\ 34 & 2.9 & 14.2\end{array}$

$\begin{array}{lll}30 & 2.5 & 16.7\end{array}$

$\begin{array}{lll}26 & 2.2 & 18.9\end{array}$

$\begin{array}{lll}23 & 2.0 & 20.9\end{array}$

$\begin{array}{lll}22 & 1.9 & 22.8\end{array}$

$\begin{array}{lll}21 & 1.8 & 24.5\end{array}$

$\begin{array}{lll}20 & 1.7 & 26.2\end{array}$

$\begin{array}{lll}19 & 1.6 & 27.8\end{array}$

$\begin{array}{lll}18 & 1.5 & 29.4\end{array}$

$\begin{array}{lll}16 & 1.4 & 30.7\end{array}$

$\begin{array}{lll}15 & 1.3 & 32.0\end{array}$


a atenção primária betinense em todas as faixas etárias (Quadro 1). Se considerarmos a evolução por faixa etária, os motivos relacionados ao sistema respiratório foram importantes na infância, com motivos relacionados à pele e ao olho tendo maior importância até os 19 anos. Na faixa etária do adolescente, as questões relativas ao genital feminino, gravidez e planejamento familiar assumem papel preponderante.

A partir da idade adulta, passam a ser mais importantes problemas digestivos até os 39 anos, e depois circulatórios até os 59 anos. Problemas neurológicos e músculo-esqueléticos permanecem frequentes durante toda a fase adulta, sendo que estes se tornam mais importantes na primeira faixa etária do idoso. Os problemas respiratórios voltam a ser frequentes no idoso acima de 70 anos.

\section{Discussão}

A classificação pelo CIAP-2 nos permite entender melhor os motivos que levam uma pessoa a consultar-se. No presente estudo, verificou-se que 32 motivos de consultas (codificados com CIAP2) foram responsáveis por $50 \%$ da demanda espontânea; e os 51 motivos mais comuns representariam $61,7 \%$ da procura nas UBS de Betim (Tabela 1).

A codificação de acordo com capítulos da CIAP-2, gênero e faixa etária reflete algumas situações peculiares à Atenção Primária. Em primeiro lugar, o capítulo "Geral e inespecífico" representou $29,8 \%$ de todos os contatos no período estudado, e foi o mais frequente em todas as faixas etárias, um dado que ilustra que os problemas de saúde na APS se apresentam de uma maneira ainda indiferenciada, e que muitos dos sintomas inclusive não chegam a ser atribuídos a um diagnóstico específico. Esta observação é corroborada pela lista dos principais motivos de consulta (Tabela 1), na qual figuram sinais e sintomas inespecíficos - tais como febre, dor generalizada e fadiga. A partir dos 70 anos, o capítulo "Geral e inespecífico" tem papel ainda mais importante, representando mais de $40 \%$ do contato com as UBSF. Além disso, observou-se uma grande procura por serviços administrativos, com um total de quase $20 \%$ dos motivos de consulta - em especial para pedido de medicação ou para mostrar resultados de exames. Estes dados são análogos aos encontrados em Portugal por Rodrigues avaliando 7855 encontros ocorridos em um período de 3 anos $^{19}$.

Os três motivos clínicos mais frequentes fo- ram cefaleia, febre e tosse - nesta ordem. São exatamente os mesmos achados de um estudo multicêntrico com 24.000 encontros codificados com CIAP-2 na África do Sul ${ }^{20}$.

No presente estudo, o número de homens que procuram a Unidade Básica de Saúde espontaneamente é muito inferior ao número de mulheres. Tal achado é consonante à recente Política Nacional de Atenção Integral à Saúde do Homem, que objetiva facilitar e ampliar o acesso da

Tabela 2. Registro dos motivos de consultas na atenção primária segundo capítulos do CIAP-2. Betim, 2010.

\begin{tabular}{lc}
\hline \multicolumn{1}{c}{ Capítulo da CIAP-2 } & $\begin{array}{c}\text { Contatos } \\
\mathbf{n}(\%)\end{array}$ \\
\hline A - Geral e inespecífico & $363(29,8 \%)$ \\
R - Respiratório & $114(9,3 \%)$ \\
D - Digestivo & $96(7,9 \%)$ \\
L - Músculo-esquelético & $87(7,1 \%)$ \\
S - Pele & $75(6,1 \%)$ \\
N - Neurológico & $75(6,1 \%)$ \\
F - Olho & $60(4,9 \%)$ \\
K - Circulatório & $60(4,9 \%)$ \\
X - Genital feminino & $59(4,8 \%)$ \\
P - Psicológico & $56(4,6 \%)$ \\
Z - Problemas sociais & $41(3,4 \%)$ \\
U - Urinário & $39(3,2 \%)$ \\
T - Endócrino/metabólico/nutricional & $36(3,0 \%)$ \\
W - Ggravidez/parto e planejamento familiar & $28(2,3 \%)$ \\
H - Ouvido & $21(1,7 \%)$ \\
Y - Genital masculino & $7(0,6 \%)$ \\
B - Sangue, sistema hematopoiético, linfático e baço & $3(0,3 \%)$ \\
Total = & $1222(100 \%)$ \\
\hline
\end{tabular}

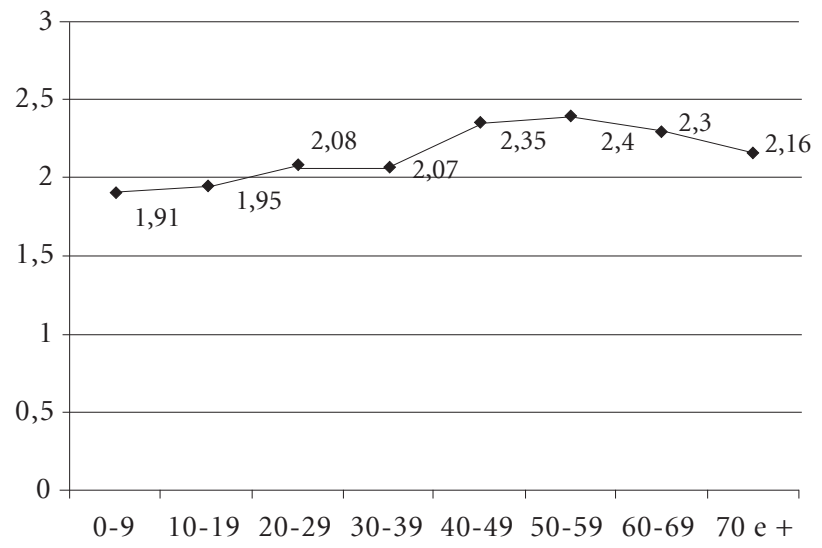

Gráfico 2. Média de motivos de consulta por paciente no atendimento a demanda espontânea. Betim, 2010. 
população masculina aos serviços de saúde - tendo em conta que a cada três mortes de pessoas adultas, duas são de homens ${ }^{21}$.

Outro dado importante é que a média de motivos de consulta das pessoas aumenta com a idade, até os 59 anos (Gráfico 2). Não se encontrou na literatura outros estudos que analisem média de motivos de consulta por faixa etária, porém entre crianças alemãs Frese encontrou médias mais baixas, variando entre 1,39 e 1,65 motivos por peso entre 0 e $19 \operatorname{anos}^{22}$. No presente estudo, estes valores variaram entre 1,91 e 1,95 - no mesmo grupo de idade. Levando em consideração todas as idades, observou-se uma média de 2,1 motivos por encontro - valor idêntico ao encontrado no estudo em Portugal ${ }^{19}$.

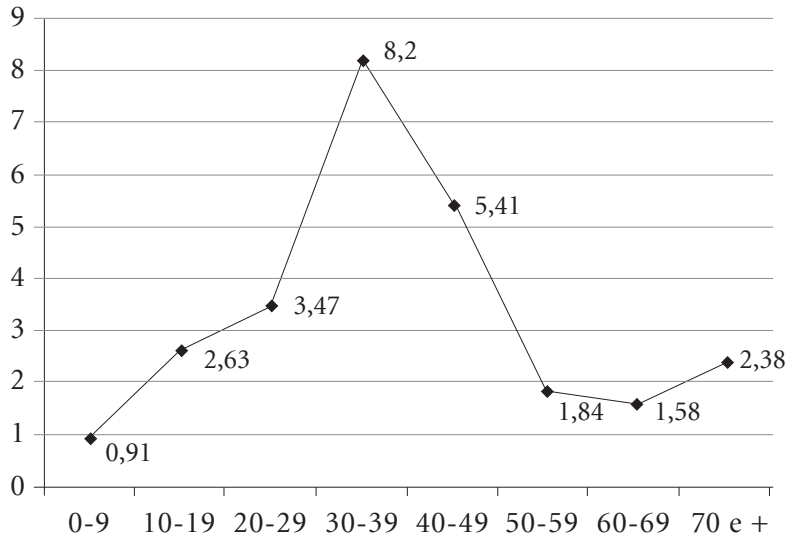

Gráfico 3. Proporção feminino vs. masculino de atendimentos a demanda espontânea. Betim, 2010.
Nas faixas etárias de 20-29 anos, 30-49 anos, e 40-49 anos não se registrou nenhum motivo de consulta relacionado ao capítulo "Genital masculino", o que pode refletir uma procura menor de homens nesta faixa (já que genital masculino somente pode ser uma queixa masculina), a não preocupação ou mesmo ausência de queixas masculinas nesta área (sinais e sintomas, contracepção, sintomas prostáticos, doenças sexualmente transmissíveis). Nestas faixas etárias registram-se as maiores diferenças entre homens e mulheres quanto a procura pelos serviços de saúde (Gráfico 3). O acesso do homem à Atenção Primária é reflexo de condições culturais e do processo de trabalho da equipe, que pode se organizar para ofertar ações em horários alternativos, visto que a maioria tem sua jornada laboral no mesmo horário de funcionamento das UBS.

Outro ponto de inflexão importante é que os capítulos "Genital Feminino" e "Gravidez, parto e planejamento familiar" foram respectivamente segundo e terceiro motivos mais comuns de consulta em mulheres na faixa etária de 10-19 anos, impactando na estatística geral como segunda e quarta causas, um indicativo importante da forte presença da sexualidade na adolescência, e suas consequências. Estes achados são semelhantes aos encontrados no estudo sul-africano, no qual Mash et al. observaram um alto número de consultas para planejamento familiar na faixa etária dos 10-14 $\operatorname{anos}^{20}$. Os serviços devem, assim, estar preparados para recepcionar estas adolescentes e ofertar opções de cuidados adequados a estas necessidades.

Analisando os capítulos mais classificados em todas as idades (Quadro 1), observa-se que den-

Quadro 1. Cinco capítulos mais frequentes de consulta de acordo com a faixa etária, Betim, 2010.

\begin{tabular}{|c|c|c|c|c|c|}
\hline $0-9$ anos & 10-19 anos & \multicolumn{2}{|c|}{$20-29$ anos } & 30-39 anos & $40-49$ anos \\
\hline $\begin{array}{c}\text { Geral e Inespecífico } \\
\text { Respiratório } \\
\text { Pele } \\
\text { Digestivo } \\
\text { Olho }\end{array}$ & $\begin{array}{c}\text { Geral e Inespecífico } \\
\text { Olho }^{\star} \\
\text { Genital Feminino* } \\
\text { Gravidez, Planejamento } \\
\text { Pele }\end{array}$ & \multicolumn{2}{|c|}{$\begin{array}{l}\text { Geral e Inespecífico } \\
\text { Digestivo } \\
\text { Genital Feminino } \\
\text { Neurológico* }^{\star} \\
\text { Urinário* }^{*}\end{array}$} & $\begin{array}{l}\text { Geral e Inespecífico } \\
\text { Digestivo } \\
\text { Musculoesquelético } \\
\text { Neurológico* } \\
\text { Genital Feminino* }\end{array}$ & $\begin{array}{c}\text { Geral e Inespecífico } \\
\text { Circulatório } \\
\text { Musculoesquelético } \\
\text { Neurológico } \\
\text { Digestivo }\end{array}$ \\
\hline $50-59$ anos & \multicolumn{2}{|c|}{ 60-69 anos } & & 70 e mais & Geral \\
\hline $\begin{array}{l}\text { Geral e Inespecífico } \\
\text { Circulatório } \\
\text { Digestivo } \\
\text { Musculoesquelético } \\
\text { Neurológico* }^{*}\end{array}$ & \multicolumn{2}{|c|}{$\begin{array}{c}\text { Geral e Inespecífico } \\
\text { Musculoesquelético } \\
\text { Circulatório } \\
\text { Psicológico } \\
\text { Respiratório }\end{array}$} & \multicolumn{2}{|c|}{$\begin{array}{c}\text { Geral e Inespecífico } \\
\text { Respiratório } \\
\text { Circulatório* }^{*} \\
\text { Músculo Esquelético* } \\
\text { Pele }\end{array}$} & $\begin{array}{l}\text { Geral e Inespecífico } \\
\text { Respiratório } \\
\text { Digestivo } \\
\text { Musculoesquelético } \\
\text { Neurológico e Pele }\end{array}$ \\
\hline
\end{tabular}

* capítulos com asterisco apresentaram mesma frequência e foram dispostos na ordem em que aparecem na CIAP-2 
tre os cinco mais frequentes, quatro deles coincidem com os dados da África do $\mathrm{Sul}^{20}$. O único que não figura na lista é o capítulo de problemas circulatórios. Em nosso estudo, este capítulo foi apenas a sétima causa geral, e sua importância dentre os motivos de consultas aumenta com a idade, sendo o segundo mais importante entre 4049 anos, 50-59 anos e a terceiro a entre 60-69 anos. A divergência em relação a outros estudos pode ser explicada pela metodologia destes, que trabalham com o volume total de pacientes enquanto optamos por avaliar os motivos de consultas que se apresentam na demanda espontânea.

$\mathrm{Na}$ infância, as queixas respiratórias assumem um papel importante, sendo este o segundo motivo mais frequente de consultas na faixa etária de $0-9$ anos, responsável por $27,3 \%$ do total de atendimentos. Este volume na infância faz com que o capítulo Respiratório seja a segunda causa geral, pois os motivos de consulta referentes a este só voltam a estar entre os cinco mais importantes no idoso. Isto está de acordo com a literatura, na qual se observa tosse (R05) entre os motivos de consulta mais comuns naqueles países onde sistematicamente se coletam dados sobre motivos de consulta com CIAP ${ }^{19-20,23-24}$.

Alguns estudos brasileiros avaliaram os motivos de consulta na Atenção Primária, sob métodos de classificação distintos, e em diferentes aspectos. Existem autores que estudaram os principais motivos de busca por atendimento, porém classificando os contatos por doenças diagnosticadas (CID-10) e não pelos motivos de consulta explicitados na CIAP ${ }^{25}$.

Há poucos estudos brasileiros utilizando a Classificação Internacional de Atenção Primária ${ }^{26}$. Dois grupos utilizaram a sua primeira edição: na década de 80, conduziu-se um teste de abrangência e exequibilidade em seis unidades de São Paulo e Rio Grande do Sul ${ }^{27}$. Outro, conduzido na Unidade de Atenção Primária Sanitária Murialdo (SSMA), na periferia de Porto Alegre, utilizou a primeira edição da CIAP (CIAP-1) para classificar a razão de 4.319 consultas ocorridas entre 1985-86, encontrando entre principais motivos de consulta a busca por motivos administrativos - dados similares aos de Betim. Neste trabalho, os 20 códigos da CIAP-1 mais frequentes explicaram $63 \%$ dos motivos de encontros ${ }^{28}$.

Finalmente, um importante estudo de atendimentos na atenção primária foi realizado em Florianópolis utilizando a CIAP-2, no qual médicos voluntários em diversas UBS registraram motivos de consulta e diagnósticos durante uma semana em cada estação do ano ${ }^{13}$. O autor ob- servou que houve pouca variabilidade, tanto nos motivos de consulta quanto nos problemas de saúde diagnosticados, nas distintas estações do ano, sendo que a codificação "medicina preventiva" e "manutenção da saúde" foram os motivos por 5 a 7\% dos atendimentos de acordo com a estação. De um total de 5.590 atendimentos, o capítulo Geral e Inespecífico respondeu por $17,1 \%$ dos atendimentos em APS, seguido de músculo-esquelético, circulatório, respiratório e digestivo. Especificamente para o período da primavera, de 1585 formulários preenchidos, as cinco causas de consulta mais comuns foram medicina preventiva/manutenção de saúde (5,5\%), medicação/prescrição/renovação/injeção referente ao aparelho circulatório $(3,1 \%)$, tosse $(3,0 \%)$, hipertensão sem complicações $(3,0 \%)$ e cefaleia $(2,8 \%)$. Novamente destaca-se que foram considerados todos os atendimentos em uma semana típica em cada estação, e não apenas os atendimentos de demanda espontânea.

É sabido que, dentre os usuários do SUS, há predomínio de mulheres, crianças, pretos e pardos, baixa escolaridade e renda ${ }^{29-30}$. A busca pelo entendimento da demanda espontânea através dos motivos de consulta - elemento-chave da CIAP - pode oferecer um maior conhecimento sobre como e porque essa população utiliza os serviços de saúde, possibilitando ampliar o acesso e responder à demanda de uma maneira centrada na pessoa.

\section{Limitações e Pontos Positivos}

Nosso estudo apresenta algumas limitações e também alguns pontos positivos. Dentre as limitações, poderíamos destacar alguns aspectos relativos à codificação. Em primeiro lugar, se trata de uma base de dados secundária, onde médicos residentes coletaram dados de seus cadernos de acolhimento, e depois os classificaram. As codificações tiveram baixo grau de concordância simples com aquela realizada pelo supervisor (ao redor de 60\%). De toda maneira, ao final foram consideradas aquelas realizadas pelo supervisor, nos casos em que ocorreram disparidades.

Uma questão a ser comentada diz respeito à sazonalidade. Este estudo foi conduzido durante a primavera e, mesmo que um outro autor ${ }^{13}$ não tenha encontrado grandes variabilidades sazonais nos motivos de consulta, alguns sintomas mais frequentes no inverno (como tosse, por exemplo) podem estar subestimados.

Outro ponto relaciona-se à amostragem por conveniência. $\mathrm{O}$ fato de a amostra não haver sido 
aleatorizada e não ser respresentativa do município impede fazer inferências a outras populações.

Apesar disso, o presente estudo apresenta resultados similares aos encontrados em outras partes do mundo, e vem acrescentar algumas qualidades metodológicas ao contexto da APS brasileira, a saber: (1) a utilização de base de dados relativa exclusivamente a demanda espontânea, via acolhimento e (2) a classificação por profissionais especificamente treinados no uso da CIAP-2.

\section{Conclusão}

A CIAP-2 se apresenta como a ferramenta mais adequada para classificar motivos de consultas na APS, pois foi desenvolvida para este contexto, e permite avaliar o motivo da consulta de acordo com a necessidade do paciente, tendo íntima relação com o método clínico centrado na pessoa. $\mathrm{O}$ fato das doenças se apresentarem ainda indiferenciadas neste âmbito de atenção torna ainda mais importante sua adoção, em especial como parte do processo de trabalho da equipe no primeiro contato ao usuário, utilizando a ferramenta do acolhimento.

A partir do entendimento dos motivos de consultas de acordo com gênero e faixa etária, a equipe pode se organizar melhor para programar as portas de saída do acolhimento, objeti- vando contemplar as necessidades de saúde da população ao lidar com a demanda espontânea.

\section{Colaboradores}

GAP Landsberg, JLS Nascimento, JMR Freitas participaram da concepção e do delineamento. AB Souza, JLS Nascimento e JMR Freitas participaram da coleta de dados. GAP Landsberg, LCM Savassi e R Azagra participaram da análise e interpretação dos dados. GAP Landsberg, LCM Savassi, AB Souza e R Azagra participaram da redação do artigo e da aprovação da versão a ser publicada.

\section{Agradecimentos}

A Gustavo Gusso e Kees Van Boven, pelas orientações e referências. A Juan Carlos Martín, pela colaboração com as análises estatísticas.

Este estudo foi apresentado como trabalho de conclusão do mestrado em Atenção Primária à Saúde da Universitat Autònoma de Barcelona, Espanha. 


\section{Referências}

1. McWhinney IR. Manual de Medicina de la Familia y Comunidad. 3a Edición. Porto Alegre: Artmed; 2010.

2. Informe de la Conferencia Internacional sobre Atención Primaria, Alma-Ata, URSS, 6-12, septiembre de 1978. Ginebra: OMS; 1978.

3. World Organization of National Colleges Academies and Academic Associations of General Practitioners/Family Physicians. Classificação Internacional de Atenção Primária (CIAP 2) - Comitê Internacional de Classificação da WONCA. Florianópolis: Sociedade Brasileira de Medicina de Família e Comunidade; 2009.

4. Meads S. The WHO Reason for Encounter classification. WHO Chronicle 1983; 37(5):159-162.

5. Lamberts H, Meads S, Wood M. Classification of reasons why persons seek primary care: pilot study of a new system. Public Health Reports 1984; 99(6):597-605.

6. Lamberts H, Meads S, Wood M. Results of the international field trial with the Reason for Encounter Classification (RFEC). Med Social Preventive 1985; 30(2):80-87.

7. Lamberts H, Wood M. International Classification of Primary Care (ICPC). Oxford: Oxford University Press; 1987.

8. Lamberts H, Hofmans-Okkes IM. Episode of Care: A Core Concept in Family Practice. J Fam Pract 1996; 42(2):161-167.

9. Okkes IM, Oskam SK, Lamberts H. The probability of specific diagnoses for patients presenting with common symptoms to Dutch family physicians. $J$ Fam Pract 2002; 51(1):31-36.

10. Crombie DL. Diagnostic process. J Coll Gen Pract 1963; 6:579-589.

11. Sampaio, LFR. O Papel das Secretarias Estaduais de Saúde no Acompanhamento e Avaliação da Atenção Primária à Saúde: Contribuições para Discussão. In: Conselho Nacional de Secretários de Saúde. Acompanhamento e avaliação da Atenção Primária. Brasil. Conselho Nacional de Secretários de Saúde. Brasília: Conass; 2004. p. 25-41. (CONASS Documenta, 7)

12. Takeda S. Organização de serviços de Atenção Primária à saúde. In.: Duncan BB, Schmidt MI, Giugliani ERJ, organizadores. Medicina ambulatorial: Condutas de Atenção Primária baseadas em evidências. 3a Edição. Porto Alegre: Artmed; 2004. p. 76-78.

13. Gusso GDF. Diagnóstico de Demanda em Florianópolis utilizando a Classificação Internacional de Atenção Primária: $2^{a}$ Edição $(C I A P$ - 2) [tese]. São Paulo (SP): Faculdade de Medicina da Universidade de São Paulo; 2009.

14. Starfield B. Atenção primária: equilíbrio entre necessidades de saúde, serviços e tecnologia. Brasília: Unesco, Ministério da Saúde; 2002.

15. George A, Rubin G. Non-attendance in general practice: a systematic review and its implications for access to primary health care. Family Practice 2003; 20: 178-84.

16. Murray M. Patient care: access. BMJ 2000; 320 (7249): 1594-1596.
17. Franco TB, Bueno WS, Merhy EE. O acolhimento e os processos de trabalho em saúde: $\mathrm{O}$ caso de Betim, Minas Gerais, Brasil. Cad Saude Publica 1999; 15(2):345-353.

18. Minas Gerais. Secretaria Estadual de Saúde [homepage na internet]. Minas adota o protocolo de Manchester na atenção primária. [acessado 2012 mar 3]. Disponível em: http://www.saude.mg.gov.br/noticias_ e_eventos/minas-adota-o-protocolo-de-manchesterna-atencao-primaria/

19. Rodrigues JG. Porque consultam os utentes o seu médico de família? Rev Port Clín Geral 2000; 16(4): 442-452.

20. Mash B, Fairall L, Adejayan O, Ikpefan O, Kumari J, et al. A Morbidity Survey of South African Primary Care. PLoS ONE 2012; 7(3):e32358.

21. Brasil. Ministério da Saúde (MS). Secretaria de Atenção à Saúde. Departamento e Ações programáticas e Estratégicas. Política Nacional de Atenção Integral à Saúde do Homem (Princípios e Diretrizes). Brasília: MS; 2008.

22. Frese T, Klauss S, Herrmann K, Sandholzer H. Children and Adolescents Reasons for Encounter. J Clin Med Res 2011; 3(4):177-182.

23. Okkes IM, Oskam SK, Van Boven K, Lamberts H. EFP. Episodes of care in Dutch Family Practice. Epidemiological data based on the routine use of the International Classification of Primary Care (ICPC) in the Transition Project of the Academic Medical Center/University of Amsterdam (19852003). In: Okkes IM, Oskam SK, Lamberts H, editors. ICPC in the Amsterdam Transition Project. CDRom. Amsterdam: Academic Medical Center, University of Amsterdam, Department of Family Medicine; 2005

24. Soler JK, Okkesb I, Oskamc S, Bovend Kv, Zivotice P, Jevticf M, Dobbsa F, Lambertsc H. An international comparative family medicine study of the Transition Project data from the Netherlands, Malta, Japan and Serbia. An analysis of diagnostic odds ratios aggregated across age bands, years of observation and individual practices. Family Practice [periódicos na internet]. Fev 2012. [acessado 2012 abr 23]. Disponível em: http://fampra.oxfordjournals.org/ content/ early/2012/02/03/fampra. cmr098.abstract

25. Carvalho MS, D’Orsi E, Prates EC, Toschi WDM, Shiraiwa T, Campos TP, Ell E, Garcia NL, Junqueira AP, Serrão SA, Tavares EL. Demanda Ambulatorial em Três Serviços da Rede Pública do Município do Rio de Janeiro, Brasil. Cad Saude Publica 1994; 10(1):17-22.

26. Sampaio MMA, Coeli CM, Almeida MTCGN, Moreno $\mathrm{AB}$, Camargo Júnior KR. Revisão sistemática do desenvolvimento e dos usos da Classificação Internacional de Atenção Primária. Cad Saúde Colet 2012; 20(1):3-14.

27. Lebrão ML. Classificação Internacional de Motivos de Consulta para Assistência Primária: Teste em algumas áreas brasileiras. Rev Saude Publica 1985; 19(1):69-78. 
28. Radaelli SM, Takeda SMP, Gimeno LID, Wagner MB, Kanter FJ, Mello VM, Borges JC, Ducan BB. Demanda de serviço de saúde comunitária na periferia de área metropolitana. Rev Saude Publica 1990; 24(3):232-240.

29. Tomasi E, Facchini LA, Thumé E, Piccini RX, Osorio A, Silveira DS, Siqueira FV, Teixeira VA, Dilélio AS, Maia MFS. Características da utilização de serviços de atenção básica à saúde nas regiões Sul e Nordeste do Brasil: diferenças por modelo de atenção. Cien Saude Colet 2011; 16(11):4395-4404.

30. Ribeiro MCSA, Barata RB, Almeida MF, Silva ZP. Perfil sociodemográfico e padrão de utilização de serviços de saúde para usuários e não-usuários do SUS - PNAD 2003. Cien Saude Colet 2006; 11(4):10111022.

Artigo apresentado em 30/04/2012 Aprovado em 17/07/1012

Versão final aprovada em 30/08/2012 